unwesentlich und daher erwähnenswert ist noch, daß auf die Zuckereinläufe der Dickdarm meist mit einer verstärkten Sekretion reagiert. Ganz gesetzmäßig scheint diese Verdünnungssekretion bei Traubenzuckerklysmen zu sein (in Versuch I und II: auf Igo bzw. $200 \mathrm{ccm}$ Einlaufsflüssigkeit 236 bzw. $230 \mathrm{ccm}$ Stuhlflüssigkeit). Dieselben Verhältnisse zeigt zwar auch der Nährzuckerversuch Nr. III (auf $200 \mathrm{ccm}$ Injektions-, $266 \mathrm{ccm}$ Stuhlflüssigkeit). Im 2. Nährzuckerfall (Versuch IV) jedoch blieb diese Wirkung aus; hier wurden auf eine Injektion von $200 \mathrm{ccm}$ Nährzuckerlösung in toto nur I $33 \mathrm{ccm}$ Stuhlflüssigkeit wieder entleert.

Als Hauptergebnis dieser 4 Versuche ist aber wohl anzusehen, daß von den Traubenzuckerlösungen in beiden Versuchen $20 \%$, von den Nährzuckerlösungen $61 \mathrm{bzw} .66 \%$ der instillierten Menge aufgenommen wurden. Möglich, da $B$ bei den Dextroseversuchen diese Werte noch etwas zu hoch gegriffen sind, da vielleicht ein gewisser Teil durch bakterielle Zersetzung sowohl der Resorption als auch der quantitativen Bestimmung im Stuhl entgangen ist. Groß kann sicherlich dieser Fehler nicht sein, sonst hätte der Geruch der Stühle eine gewisse Säuerung verraten müssen, auch wäre sonst wohl das Ergebnis beider Versuche nicht so gleichmäßig ausgefallen. Bei den Nährzuckerversuchen spielt die genannte Fehlerquelle bestimmt nur eine geringe Rolle, da ja hier, worauf schon v. Noorden hinweist, die rasche Resorption des aus Dextrin langsam entstehenden Zuckers eine stärkere Gärung sehr unwahrscheinlich macht. Im Gegenteil, den Verhältnissen in der Praxis gegenüber sind diese Werte wohl noch zu gering bemessen. Denn die Untersuchung in der Stoffwelchselschwebe bietet ungünstigere Bedingungen, als sonst die Praxis; werden doch erfahrungsgemäß die Einläufe bei Seiten- oder auch Bauchlage der Kinder viel leichter gehaiten.

Wenn in den vorigen Nährzuckerversuchen den Kindern von eingeführten $36 \mathrm{~g}$ reduzierender Substanz $22 \mathrm{~g}$ zugute kommen, so gelten diese Zahlen nicht ohne weiteres für Säuglinge mit erhöhter Peristaltiik. Schon die infolge Gärung der Resorption entgehende Zuckermenge ist hier, selbst wenn ein Dextrin enthaltendes Kohlenhydrat verwandt wird, ein wenig höher einzuschätzen. Auch sonst hat eine quantitative Bestimmung der aufgenommenen Kohlenhydrate bei einem solchen Kinde nur bedingten Wert, da ja hier, worauf schon oben hingewiesen wurde, die mehr oder weniger starke Intensität des Durchfalls eine große Rolle für das ,Halten" der Klistiere spielt.

Um uns aber wenigstens ungefähr $\mathrm{über}$ die einem derartigen Kinde durch die Nährzuckerklistiere zugeführte Energiemenge $\mathrm{zu}$ unterrichten, haben wir in einem solchen Fall eine quantitative. Untersuchung vorgenommen:

Schwere akute Dyspepsie mit Gewichtsabfall in wenigen Tagen um etwa $300 \mathrm{~g}$. Im Harn kein Zucker nachweisbar. Nach I2stündiger Teediät erhält das Kind kleine Mengen Fravenmilch und Buttermilch. Am Tage darauf werden ihm in 5 stündigen Abständen 3 Klistiere von $100 \mathrm{ccm}$ Io proz. Nährzuckerlösung mit Opiumvorklistier und Opiumzusatz verabreicht; peroral erhält es an diesem Tage: Io $\times$ i $5 \mathrm{Br}$. M., io $\times 5 \mathrm{Br}$. M.

Das Ergebnis ist ein relativ günstiges:

\begin{tabular}{c|c|c|c|c}
\hline Eingeführt & $\begin{array}{c}\text { Stuh1 und } \\
\text { Schlauch }\end{array}$ & Harn & Retention \\
absolut & $\begin{array}{c}\text { in } \% \text { der eingeführten } \\
\text { Menge }\end{array}$ \\
\hline 26,7 & 10,5 & 0,095 & $16, \mathrm{~g}$ & $\begin{array}{c}60 \% \text { reduzierende } \\
\text { Substanz }\end{array}$
\end{tabular}

Von $30 \mathrm{~g}$ Nährzucker $=27 \mathrm{~g}$ reduzierender Substanz werden $\mathrm{I} 6 \mathrm{~g}=60 \%$ aufgenommen. Das entspricht einer Energiemenge von 65 Calorien. Hätten wir die Anzahl der Klistiere, was in diesem Falle nicht erforderlich, aber sehr wohl möglich war, auf 5 erhöht, hätten wir auch statt der ro proz. eine 20 proz. Lösung verwandt*), so wären wir trotz des Durchfalls noch auf weit höhere Energiemengen gekommen. Die Verdünnungssekretion, die bei der starken Reizbarkeit

*) Im allgeminen werden die ro prozentigen Lösungen besser gehalten; sehr häufig zeigt der dyspeptische Darm jedoch auch den so prozentigen gegenüber eine große Toleranz. des dyspeptischen Darms sicherlich nicht gering war, kam wahrscheinlich infolge einer in diesem Falle ganz enorm raschen Resorption und Rückresorption - überhaupt nicht zur Geltung. Während $300 \mathrm{ccm}$ Flüssigkeit instilliert wurden, wurden nur $82 \mathrm{ccm}$ mit dem Stuhle wieder entleert.

Endlich suchten wir uns noch auf einfache klinische Weise von dem Nutzeffekt der rectal eingeführten Kohlenhydrate zu überzeugen. Das mußte leicht gelingen, wenn wir zunächst eine kalorisch ausreichende Nahrung mitwenig Kohlenhydraten darreichten und darauf das Kohlenhydrat in Gestalt der Zuckerklistiere superponierten. Der durch die Kohlenhydrateinführung erzeugte Nutzeffekt mußte in solchem Falle an dem prompt einsetzenden Gewichtsanstieg zutage treten. Die klinische Beobachtung zeigte, daß diese Kohlenhydratwirkung in vollem Umfange eintrat.

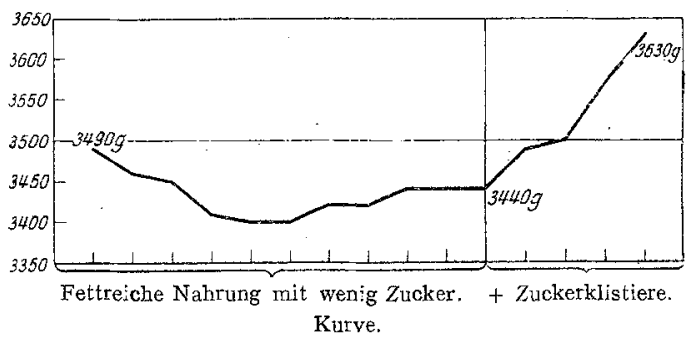

Ein fettreich und kohlenhydratarm (Eiweißmilch mit $5 \%$ Butter) ernährter Säugling war im Gewicht mehrere Tage ganz stillgestanden, nun erfolgte die Kohlenhydratzulage in Gestalt der Zuckerklistiere (5 Nährzuckerklysmen 20 proz. $z u$ je $100 \mathrm{~g}$ ). Sofort wurde die rectale Kohlenhydratzufuhr mit einem Gewichtsanstieg von $200 \mathrm{~g}$ in 4 Tagen beantwortet, ein sicheres Zeichen für die Verwertung des so eingeführten Kohlenhydrats.

Quantitative Untersuchung und klinische Beobachtung zeigten demnach, daß es gelingt, bemerkenswerte Mengen von Kohlenhydraten rectal zu verabreichen und derVerwertung im Stoffwechsel zuzuführen.

In Fällen, in denen sich die orale Zufuhr von Kohlenhydraten aus äußeren (Pylorusstenose) oder inneren Gründen (Durchfälle des Atrophikers) verbietet, ist daher die Anwendung der Zuckerklistiere angezeigt, um wenigstens einen Teil der schweren Gefahren des kompletten Hungers abzuwenden.

Li te r a t u r: ${ }^{1}$ ) Göpper., Monatsschr. f. Kinderheilk. 1920. 2) v. NoOrden-SAlomon, Alloem. Diätetik. Ig20, - ${ }^{3}$ ) Strauss Charité-Annalen 22. I902. - ${ }^{4}$ ) BINGEI, Therap. d. Gegenw. 1905.5) REACH, Arch. f. exp. Pathol. u. Pharmalool. 47. 1902. - 6) PLATENGA, Der Wert der Nährklistiere, Inaug.-Diss., Freiburg. i. Br. I 898. - ${ }^{7}$ ) v. Halasz, Arch. f. klin. Med. 98 igio. - 9 ) JACOBSOHNREwald, Therap. d. Gegenw. I9I.I. - $\left.{ }^{9}\right)$ Schönborn, Zur Frage der Resorption von Kohlenhydraten im menschlichen Rectum, Inaug.-Diss., Würzburg I 897. - ${ }^{10}$ ) Scrmidi, Münch. med. Wochenschrift 1903, S. 2054

\section{EINE WESENTLICHE VERBESSERUNG DER DURCHLEUCHTUNGSTECHNIK DER LUNGENSPITZEN.}

\section{Dr. KARL FRIK.}

Aus der L. med. Klinik der Univers. Bejlin. (Direktor: Prot. W. HIS.)

Die unerschwinglichen Preise für Röntgenplatten zwingen uns jetzt oft, uns mit einer Durchleuchtung zu begnügen, wo wir früher glaubten, ohne Aufnahme nicht auskommen zu können. Wir müssen daher mehr als je bestrebt sein, unsere Durchleuchtungstechnik $z u$ vervollkommnen; jedes wirksame Mittel hierzu ist zu begrüßen.

Ich gebe im folgenden einen kleinen Kunstgriff bekannt, der es gestattet, die Lungenspitzen in vielen Fällen ganz erheblich viel klarer zu sehen, als es ohne ihn möglich wäre. Der Kunstgriff ist so einfach, daß ich nicht annehmen kann, es sei bis jetzt außer mir keiner auf den Gedanken gekommen, 
ihn anzuwenden. Ich finde ihn aber nirgends in der Literatur erwähnt, und gelegentlich seiner Demonstration in der Berliner Röntgenvereinigung ${ }^{\mathbf{1}}$ ) stellte sich heraus, da.B er in der Tat offenbar nicht bekannt ist. Auch vielfache Erfahrung in Arztekursen hat mir bestätigt, daß $\beta$ die Praktiker - und solche haben ja jetzt vielfach eigene Röntgenapparate - den Kunstgriff nicht kennen.

Bei der gewöhnlichen dorso-ventralen Durchleuchtung der Lungen sieht man oft den medialen Teil der Spitzenfelder in mehr oder weniger großer Ausdehnung stark homogen

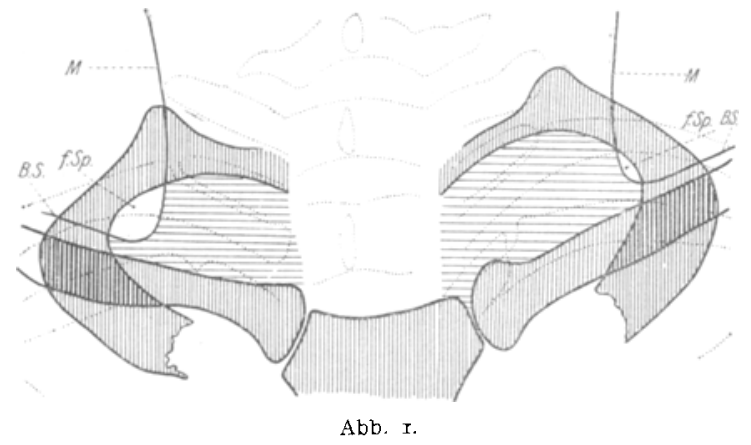

Senkrech sehraficht: Die die Spitzenfelder umrahmenden Knochen: I. Rippe, Schlüsselbein und Manubr. sterni. Wagrecht schraffiert: Der vom Musc. sterno-cleido-mast. verschleierte Teil der Spitzenfelder.

........ Wirbel, II. und III. Rippe.

$M$ : Muscul. sterno-cleido-mast.

f. Sp.: Von letzterem freigelassene Stelle des Spitzenfeldes.

B. S.: Begleitschatten des Schlüsselbeines (Haut).

verschleiert. Die laterale Grenze der Verschleierung ist scharf, verläuft nahezu senkrecht von oben nach unten und geht unten in scharf gezeichneter Kurve in den oberen Rand des von der Haut herrührenden Begleitschattens des Schlüsselbeins über. Wenn alle genannten charakteristischen Eigenschaften des Schattens deutlich ausgesprochen sind, dann

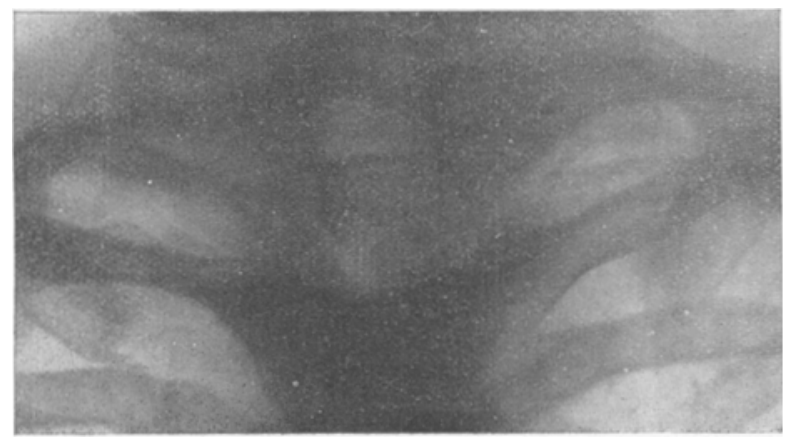

Abb. ra.

Originalbild zu Abb. I.

ist kein $Z_{\text {weifel }}$ es handelt sich um den Schatten des Musculus sterno-cleido-mastoideus (vgl. Abb. I und ra).

Oft ist aber die lateraie Grenze des Schieiers nicht deutlich zu erkennen oder verliert sich völlig im Rippenschatten, oft sieht man auch nicht ihren Ubergang in den Begleitschatten des Schlüsselbeins. Steht außerdem der Patient auch nur ein wenig nach einer Seite gedreht, dann kann das eine Spitzenfeld vollkommen, vom anderen nur ein kleines mediales Stück durch den Musculus sterno-cleido-mastoideus verschleiert.sein. Dann ist oft die wahre Natur des Schleiers auf den ersten Blick gar nicht zu erkennen. In allen Fällen ist zu versuchen, den Schleier durch Wegdrängen des Musculus sterno-cleido-mastoideus zu beseitigen. Welch überraschenden Erfolg man damit haben kann, zeigt Abb. 2 und 2a. Die

1) S:tzung v. 23. III. rg22. Vgl. Fortschr. a. d. Geb. d. Röntgenstr. Bd. XXIX, F. 3. S. 371 .
Technik ist folgende: Man umgreift, die Hand im Schatten des Patienten zwischen ihm und dem Schirm nach oben schiebend, mit dem Endglied des Zeigefingers den lateralen Rand des Musculus sterno-cleido-mastoideus, wobei man darauf achten mußB, daß man tatsächlich den Muskel selbst und nicht nur die darüber liegende Haut faßt, und drängt den Muskel kräftig medianwärts; den Patienten hält man mit der anderen Hand fest, damit er nicht dem Druck ausweicht. Das von dem Schatten des Muskels befreite Spitzenfeld ist nun stark aufgehellt und man kann darin auch zarte Schatten-

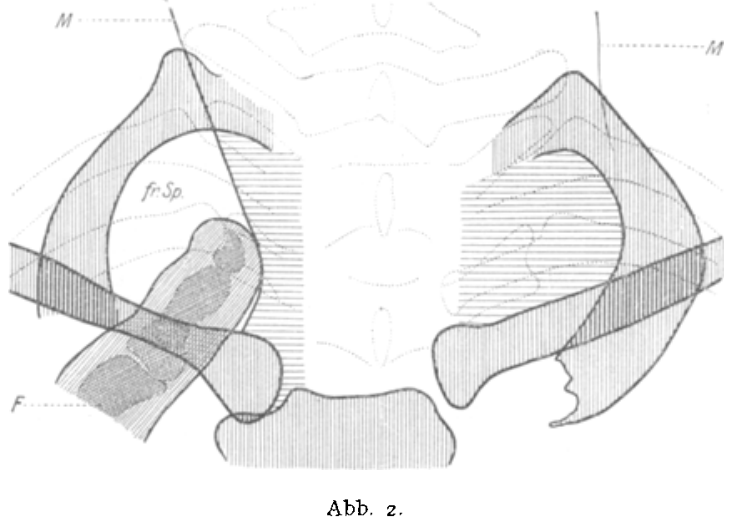

Rechter Sternocleidomast. mittels Finger medianwärts gedrängt.

Schraffierung siehe oben Abb. I.

$M$ : Musc. sterno-cleido-mast.

$F$ : Finger des Untersuchers.

fr. Sp.: Freies Spitzenfeld (das andere ist durch ren Musc. sterno-cleido-mast. völlig verschleiert).

herde erkennen, die einem ohne den Kunstgriff entgangen wären. Noch wesentlich sicherer wird die Erkennung zarter Schattenherde, wenn man außerdem den Patienten leicht hin und her dreht, so daß die Schatten durch die Interkostalräume huschen, denn bewegte Schatten fallen immer leichter in die Augen, als stillstehende. So groß, wie in dem in Abb. 2

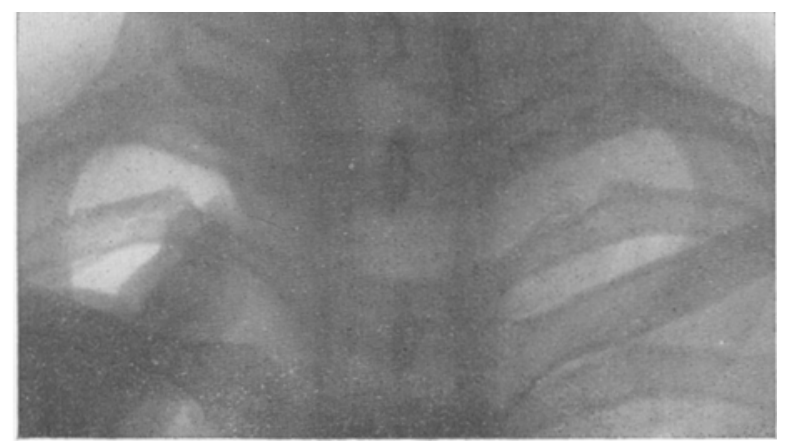

Abb. 2a.

Originalbild $z$ a Abb, 2. Derselbe Fatient wie Abb. I und I a.

abgebildeten, ad hoc ausgesuchten Fall, ist der Unterschied zwischen dem durch die leichte Drehung des Kopfes völlig vom Musculus sterno-cieido-mastoideus verschleierten und dem durch Wegdrängen des Muskels aufgehellten Spitzenfeld nicht immer, aber immer bekommt man eine deutliche Aufhellung durch den Kunstgriff, den man in keinem Falle anzuwenden versäumen sollte.

Zur Schonung der eigenen Hand kann man den Zeigefinger auch durch ein Instrument ersetzen; ich habe dazu z. B. den einen Arm eines Tasterzirkels benutzt; aber für den Patienten ist der Finger angenehmer. Leider stellen sich die Patienten meist sehr ungeschickt an, wenn man sie auffordert, den Griff selbst zu machen. 